Elsevier Editorial System(tm) for International Journal of Psychophysiology Manuscript Draft

Manuscript Number: CCD2456R2

Title: Startle responding in the context of visceral pain

Article Type: Research Paper

Keywords: mechanical esophageal stimulation; visceral pain; startle; gender differences

Corresponding Author: Dr. Erik Ceunen, Ph.D

Corresponding Author's Institution: University of Luxembourg

First Author: Erik Ceunen, Ph.D

Order of Authors: Erik Ceunen, Ph.D; Jonas Zaman, MSc; Natacha Herssens, MSc; Lukas Van Oudenhove, PhD; Huynh Giao Ly, PhD; Steven J Coen, PhD; Jan Tack, PhD; Johan W. S Vlaeyen, PhD; Ilse Van Diest, $\mathrm{PhD}$

Abstract: This study aimed to investigate affective modulation of eye blink startle by aversive visceral stimulation. Startle blink EMG responses were measured in 31 healthy participants receiving painful, intermittent balloon distentions in the distal esophagus during 4 blocks (positive, negative, neutral or no pictures), and compared to startles during 3 'safe' blocks without esophageal stimulations (positive, negative or neutral emotional pictures). Women showed enhanced startle during blocks with distentions (as compared to 'safe' blocks), both when the balloon was in an inflated and deflated state, suggesting that fear and/or expectations may have played a role. Men's startle did not differ between distention and non-distention blocks. In this particular study context affective picture viewing did not further impose any effect on startle eye blink responses. The current results may contribute to a better understanding of emotional reactions to aversive interoceptive stimulation. 
ONS KENMERK

UW KENMERK LEUVEN,

$10 / 07 / 2015$

\section{Re: $\quad$ Startle responding in the context of visceral pain}

Dear Editor,

We are submitting a second revision of our manuscript "Startle responding in the context of visceral pain" for publication in International Journal of Psychophysiology.

The present work is original as it has not been published before. It is not submitted for publication elsewhere, and will not be submitted elsewhere now that the editiorial team of the International Journal of Psychophysiology has recommended publication. The final minor changes that were recommended prior to publication, have now been implemented.

We would like to thank you for having collected the thougthful reviews for our work and for allowing us to provide a meaningful contribution to the International Journal of Psychophysiology.

Your sincerely,

Erik Ceunen,

also on behalf of the co-authors 
Dear Editor,

Thank you for collecting these final minor, but very helpful suggestions. Please find below the changes we made as marked in blue.

Best Regards,

Erik Ceunen, also on behalf of the coauthors.

Ms. Ref. No.: CCD2456R1

Title: Startle responding in the context of visceral pain

International Journal of Psychophysiology

Dear Dr. Ceunen,

Your paper, entitled, "Startle responding in the context of visceral pain," has been reviewed for publication in the International Journal of Psychophysiology. The editorial team has recommended publication, but has requested some minor revisions to your manuscript.

Please see the comments appended below to guide you in preparing your revision. Please submit a list of changes or a rebuttal against each of the points noted in the reviews along with the revised manuscript.

Because we are trying to facilitate timely publication of manuscripts submitted to the International Journal of Psychophysiology, your revised manuscript should be uploaded as soon as possible but no later than 30 days.

Once you have completed your revisions, kindly resubmit your manuscript as a revised paper via the Elsevier Editorial System (http://ees.elsevier.com/intpsy/). Please ensure that you use the option available under "Submissions Requiring Revision" on your author homepage.

Your login details are as follows: Your username is: erik ceunen@yahoo.com. If you need to retrieve password details, please go to http://ees.elsevier.com/intpsy/automail_query.asp.

We would like to thank you for contributing this interesting paper to the International Journal of Psychophysiology, and we look forward to receiving your revised manuscript.

Please note that this journal offers a new, free service called AudioSlides: brief, webcast-style presentations that are shown next to published articles on ScienceDirect (see also http://www.elsevier.com/audioslides). If your paper is accepted for publication, you will automatically receive an invitation to create an AudioSlides presentation.

Yours sincerely, 
Paul Pauli, PhD

Action Editor

International Journal of Psychophysiology

Reviewers' comments:

Reviewer \#1: Thank you for the well done changes.

I have only two minor suggestions.

-Reword the last part of the abstract (see suggestion below).

-Drop the word "affective" in the first sentence of the discussion section.

These two suggestions have now been implemented.

- For the first suggestion on rewording the last part of the abstract, we have followed the exact wording below, as suggested by the reviewer.

- The word 'affective' has been removed from the first sentence of the discussion section.

Suggested wording for the last part of the abstract:

Women showed enhanced startle during blocks with distentions (as compared to 'safe' blocks), both when the balloon was in an inflated and deflated state, suggesting that fear and/or expectations may have played a role. Men's startle did not differ between distention and non-distention blocks. In this particular study context affective picture viewing did not further impose any effect on startle eye blink responses. The current results may contribute to a better understanding of emotional reactions to aversive interoceptive stimulation.

The wording as suggested by Reviewer $\# 1$ above is now used in the abstract. 


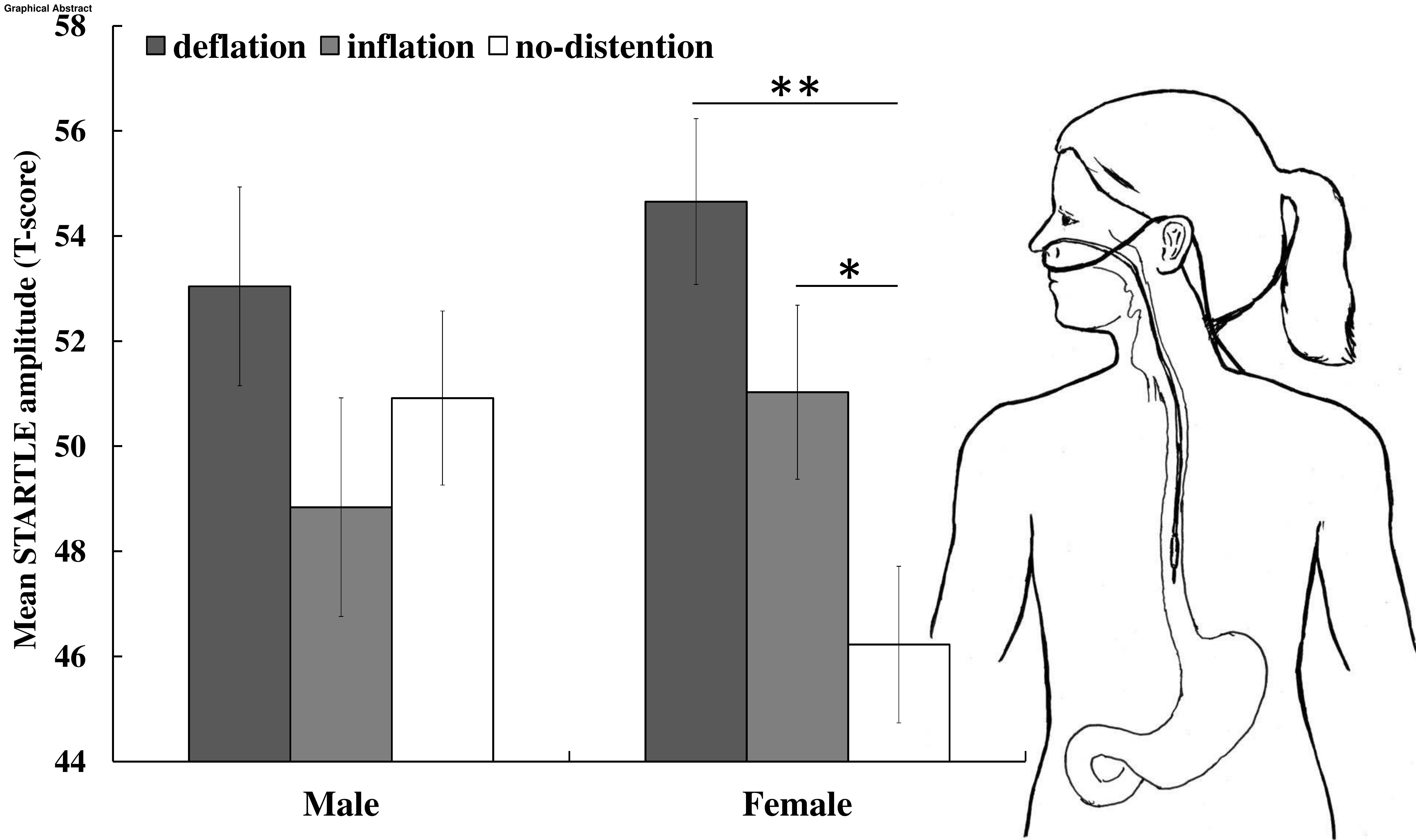




\section{Highlights}

- Startle was measured in the context of esophageal stimulation

- In blocks with intermittent stimulation, women showed startle potentiation

- Men's startle did not differ between distention and non-distention blocks

- Results suggest future studies should further look into gender differences 


\title{
Startle responding in the context of visceral pain
}

\author{
Ceunen, Erik ${ }^{\mathrm{a}}$ \\ Zaman, Jonas $^{\mathrm{a}}$ \\ Herssens, Natacha ${ }^{\mathrm{b}}$ \\ Van Oudenhove, Lukas ${ }^{\mathrm{b}}$ \\ Bogaerts, Katleen $^{\text {a }}$ \\ Ly, Huynh Giao ${ }^{b}$ \\ Coen, Steven J. ${ }^{\mathrm{b}}$ \\ Tack, Jan ${ }^{\mathrm{b}}$ \\ Vlaeyen, Johan W.S. ${ }^{\text {a }}$ \\ Van Diest, Ilse $\mathrm{a}^{\mathrm{a}}$
}

\section{Institutional Affiliations:}

a. Health Psychology, KU Leuven

b. Translational Research Center for Gastrointestinal Disorders (TARGID), Department of Clinical \& Experimental Medicine, KU Leuven

\section{* Corresponding author:}

Ilse Van Diest | Research Group Health Psychology | KU Leuven | Tiensestraat 102, box 3726 | 3000 Leuven (BELGIUM)

$\mathrm{T}+3216326029$

e-mail: $\underline{\text { Ilse.VanDiest@ppw.kuleuven.be }}$ 


\begin{abstract}
This study aimed to investigate affective modulation of eye blink startle by aversive visceral stimulation. Startle blink EMG responses were measured in 31 healthy participants receiving painful, intermittent balloon distentions in the distal esophagus during 4 blocks (positive, negative, neutral or no pictures), and compared to startles during 3 'safe' blocks without esophageal stimulations (positive, negative or neutral emotional pictures). Women showed enhanced startle during blocks with distentions (as compared to 'safe' blocks), both when the balloon was in an inflated and deflated state, suggesting that fear and/or expectations may have played a role. Men's startle did not differ between distention and non-distention blocks. In this particular study context affective picture viewing did not further impose any effect on startle eye blink responses. The current results may contribute to a better understanding of emotional reactions to aversive interoceptive stimulation.
\end{abstract}

Keywords: mechanical esophageal stimulation; visceral pain; startle; gender differences 


\section{Introduction}

Eye blink startle is modulated by affective background (Vrana et al., 1988). As such, it can be used to distinguish between appetitive, neutral, and aversive emotional states, with decreased magnitudes during the former, and increased magnitudes during the latter. This affective modulation of startle is only evident when the emotional states are sufficiently arousing or intense (Bradley et al., 2001). Such emotional and physiological arousal can be indexed by skin conductance, reflecting sympathetic activation (Dawson et al., 2007). Together, eye blink startle magnitude and skin conductance measures can be used to assess the biphasic aspects of emotion, respectively reflecting motivational direction, and motivational intensity (Bradley and Lang, 2007).

Because affective modulation of startle has been found when using visual (e.g., Jansen and Frijda, 1994; Schupp et al., 1997), auditory (Bradley and Lang, 2000), and olfactory (Ehrlichman et al., 1995) mood stimuli, it has been suggested that such modulation occurs regardless of the sensory modality used for mood induction (Bradley and Lang, 2007). Recent research with interoceptive stimuli seems to contest this notion (Ceunen et al., 2013). For example, during aversive and arousing dyspnea as induced by loaded breathing (a mechanical stimulus creating respiratory resistance similar to breathing through a straw), startle potentiation has not been evidenced (Pappens et al., 2010). Moreover, when fearinducing dyspnea was elicited by $\mathrm{CO}_{2}$ inhalation, it led to inhibition of startle, relative to startle measured during room air breathing (Pappens et al., 2012). Also during tonic cold pain and tonic heat pain, which are both interoceptive according to the definition of interoception forwarded by Craig (2002), no startle potentiation has been observed (Deuter et al., 2012; Horn et al., 2012). In contrast, during anxious anticipation of respiratory and other interoceptive sensations, the expected startle potentiation has been found (Hubbard et al., 
2011; Lang et al., 2011; Melzig et al., 2008; Naliboff et al., 2009; Pappens et al., 2013; Twiss et al., 2009).

Apart from a small number of studies, at present the pattern of startle in response to emotions induced by actual presence, rather than anticipation of interoceptive sensations (including pain), largely remains to be elucidated. Therefore, the major aim of the current study was to unveil the startle response pattern that occurs in a period of time during which there is repeated exposure to an aversive interoceptive stimulus, namely stimulation of the distal esophagus at pain threshold, i.e. first sensation of pain. We hypothesized that startle potentiation would occur during 'unsafe' periods during which painful stimulation occasionally and unpredictably occurred relative to 'safe' periods without such stimulation.

An additional aim was to find out whether in the unsafe periods, startle potentiation is present both during anticipation of and during actual painful stimulation, relative to safe periods. Based on the various findings on startle in response to interoceptive stimuli as discussed earlier, it would be expected that startle in anticipation of visceral stimulation is elevated relative to startle elicited during actual visceral stimulation.

Although the study was not purposely set up to study gender differences, findings in the literature suggest that sex differences exist in neurobiological mechanisms involved in the processing of visceral signals (Kano et al., 2013; Kilpatrick et al., 2010; Labus et al., 2013; Pennebaker and Roberts, 1992). Therefore, we included gender in our analyses in an attempt to explore its possible effects. As the literature on startle in response to interoceptive stimuli is on itself already relatively limited, it follows that the literature on gender effects on startle during interoceptive stimulation is nearly non-existent. Therefore we did not make any specific assumptions on how the startle would be different between genders, if at all, even if there are indications for the existence of gender specific differences in the processing of interoceptive stimuli. 
The choice for distal esophageal stimulation was in part motivated by the ability to stimulate solely visceral tissue without involving stimulation of any overlying somatic tissue (Aziz et al., 2000), thus being classified as an interoceptive stimulation even by those who define interoception in its strictest sense (e.g., Dworkin, 2007). Opting for the esophagus as the site of stimulation also allows for future research to expand upon the current research findings, for example contrasting purely visceral stimulation (distal esophagus) with purely somatic stimulation (proximal esophagus) (Aziz et al., 2000). We decided to stimulate at first pain threshold for our stimulus to qualify as aversive; pain by definition comprises a component of unpleasant affect according to the International Association for the Study of Pain (Merskey and Bogduk, 1994).

Given the extensive literature of affective modulation of pain (Rhudy and Meagher, 2001; Wiech and Tracey, 2009), we included three different affective backgrounds by means of pictures. These were included in order to explore whether these backgrounds would differently affect the eye blink startles elicited during blocks with esophageal distention compared to those without. An additional argument in favor of the inclusion of the emotional picture series that we had in mind when designing the experiment, was that the inclusion of affective pictures would control for any extraneous confounding factors that might affect mood of participants.

\section{Methods}

We recruited 31 healthy university students ( 18 women); they received $50 €$ for participating in the study. As 7 participants were excluded from analysis (see Data analysis section, subheading Eye blink startle) we only studied the responses of the remaining 24 participants (14 women); they had a mean age of 22 years old $(\mathrm{SD}=3)$. All participants received an informed consent prior to deciding on whether to participate, and reread the 
consent just prior to signing it. The informed consent was in accordance with the declaration of Helsinki (1997) and stated that participants were free to halt their participation at any point without any negative consequences. This study was approved by the psychological and medical ethical committees of KU Leuven.

At the start of the experiment, a standard pediatric catheter was inserted trans-nasally with the end reaching the distal, autonomously innervated part of the esophagus, $35 \mathrm{~cm}$ from the nostril. A deflated medical balloon was firmly attached to the end positioned in the esophagus, while the extraneous part of the catheter was gently attached to the face with tape to prevent it from moving. The remaining end was draped over the ear and connected to an air filled syringe. Although the insertion itself was invariably experienced as unpleasant, once the catheter was in its proper position, we did not continue with the next steps of the procedure, until subjects reported they became habituated to any sensations due to the presence of the catheter, which never took more than a few minutes. This procedure has been used extensively in previous research (e.g., Aziz et al., 2000; Coen et al., 2009).

After inserting the catheter, the pain threshold of participants was determined by gradually inflating the esophageal balloon thrice, and taking the average of these three volumes of distention (at which subjects indicated they first felt a sensation that they would call painful) as the best approximation of their actual pain threshold. Additionally, during threshold determination, we assured ourselves that the balloon was in the distal part of the esophagus by asking participants if they could indicate where they felt a sensation: if their answer indicated they could feel the sensation somewhere around their chest level, but that they could not locate it at a specific site, this was taken to indicate the balloon was indeed in the autonomously innervated, i.e. visceral part of the esophagus (Aziz et al., 2000). After threshold determination, a 3-minute baseline measure of skin conductance was obtained, and subjects were exposed to 10 startle probes in order to habituate them before proceeding to the 
actual experiment. There was an interval of 10 seconds between each of these habituation probes.

The experiment consisted of seven blocks, each lasting 5 minutes 23 seconds. In six of the seven blocks, participants viewed a series of 36 mood-inducing pictures of one same valence, selected from the International Affective Picture System (IAPS, Lang et al., 2008). Each picture was presented only once throughout the entire experiment ( $8 \mathrm{~s}$ on, 1s off). Two picture blocks contained positive pictures, two blocks contained neutral pictures, and two blocks contained negative pictures. Both blocks of each valence had equal mean valence, arousal, and dominance levels according to the normative data collected by Mikels et al. (2005). Furthermore blocks of the same valence were also matched according to the proportion of animals, objects, humans, and overall picture content complexity (see appendix for more information of the exact pictures that were selected). A similar blocked presentation of pictures has been used earlier by Smith, Bradley and Lang (2005), and results of their study indicate that affective modulation is maintained and even increased throughout the consecutive presentation of pictures of the same valence.

In the block without pictures, participants were instructed to look at a fixation cross presented on the monitor. The latter block, as well as 3 of the picture viewing blocks (one for each picture valence) were accompanied by 10 esophageal balloon distentions. Balloon distentions in those blocks were administered manually at individual pain threshold, started simultaneously with picture onset (in the distention blocks with pictures), and ended after 5 seconds. Inflations and deflations were performed as instantaneous as physically possible, implying that throughout each distention, the balloon's volume was nearly constant. The available air volume for inflation was limited to the individual threshold to prevent accidentally exceeding the determined volume. The order of the block presentations was semi-randomized, taking into account that blocks of the same pictorial valence or blocks with 
distentions would never be presented consecutively. The first, third, fifth and seventh blocks were blocks with distentions, whereas the second, fourth and sixth block were free of distentions. Participants were informed that blocks with and blocks without distentions would alternate, and were informed that a new block would start only after filling in self-report items. The 10 distentions in each of the four distention blocks occurred with varying intervals between each distention (22-40s), making the exact onset of each distention unpredictable.

Self-reports of fear, valence and arousal were obtained after each block, respectively on a horizontal VAS ranging from 0 (no fear at all) to 10 (worst fear imaginable), and two 9point self-assessment manikin (SAM; Bradley and Lang, 1994) scales, one ranging from unpleasant (1) to neutral (5) to pleasant (9), and the other from calm (1) to aroused (9). Furthermore, participants rated pain intensity on vertical bars after each block. Scores ranged from 'no sensation' (0) to 'moderate' (5) to 'most intense I can imagine' (10). Finally, we also assessed to which extent persons had experienced a set of 10 hyperventilation symptoms on a 5 point Likert-scale. The latter self-reports addressed an exploratory research question that goes beyond the scope of the present paper and will not be further discussed here.

Per block, 10 white noise startle probes $(50 \mathrm{~ms})$ with a peak $\mathrm{dBA}$ of $103 \mathrm{~dB}$ were administered binaurally. Although startles were always administered 4 seconds after picture onset, their occurrence was made unpredictable by varying the interval between startle probes (27-54s). The EMG eye blink startle responses were measured by three $\mathrm{Ag} / \mathrm{AgCl}$ Coulbourn electrodes (V91-02, 4mm) according to the guidelines described by Blumenthal et al. (2005). A V75-04 (Coulbourn Instruments) isolated bioamplifier with a $13 \mathrm{~Hz}$ high pass, and $1 \mathrm{kHz}$ low pass bandpass filter was used to amplify the raw signal. This signal was then rectified and smoothed by a Coulbourn integrator (V76-24; time constant=20ms). Startle responses were sampled at $1000 \mathrm{~Hz}$ and were recorded starting $500 \mathrm{~ms}$ prior to probe onset until $1000 \mathrm{~ms}$ after probe onset. 
Skin conductance levels (SCLs) at the hypothenar eminence of the non-dominant hand were recorded throughout each block using a Coulbourn skin conductance coupler (V71-23) providing a constant of 0.5V across two V91-01 (8mm) electrodes. The signal was digitized at $10 \mathrm{~Hz}$.

Affect 4.0 software (Spruyt et al., 2010) and a 16-bit data acquisition card (National Instruments, Austin, Texas) were used to collect EMG and SCL data. Physiological data were organized using JMP® 9 software, further processed offline for parameter extraction with PSPHA (De Clercq et al., 2006), and analyzed using STATISTICA 10 software.

\section{Data Analysis}

3.1 Manipulation check. Pain ratings of all blocks were entered in a repeated measures ANOVA with gender as a between subject variable and block as a within subject variable (7 levels). An a priori assumption was that pain intensity would be higher during distention trials as compared to non-distention trials for both genders. To explore potential sensitization or habituation effects on pain, we also ran a repeated measures ANOVA with position of the distention block (1st, 3rd, 5th or 7th) as a within subject variable and gender as a between subject variable.

3.2 Eye blink startle. Eye blink EMG magnitudes were obtained by subtracting the mean baseline value ( 0 to $20 \mathrm{~ms}$ following probe onset) from the peak value ( 21 to $175 \mathrm{~ms}$ following probe onset). All startle responses were visually inspected and values were discarded when there was already blink activity between startle probe onset and minimal blink onset latency; this was the case in $16,9 \%$ of the cases. Four participants showed no or rejected startle response in $>66 \%$ of all startle trials; those participants were classified as non-responders and further excluded from analyses (Blumenthal et al., 2005). 
Within the distention blocks and irrespective of picture viewing, another 3 participants failed to show at least 4 valid startle responses either during actual balloon inflations, either in between distentions (deflations). In order to avoid that unreliable estimations of the startle response (based on less than 4 startles during actual inflation or deflation) would affect our findings, these participants were also excluded from analyses, resulting in a final sample of $\mathrm{N}$ $=24(14$ women $)$.

Averaged startle responses were standardized within participants (T-scores), so that they would be relative to each subjects' range of responding (see also Table 1). We averaged each participant's startle responses for 11 within subject conditions (see also Table 1). Three conditions (further called 'safe') referred to distention-free blocks of picture viewing (one positive, one neutral, one negative). Four other conditions (further called 'inflation') referred to startles during actual distention (positive, neutral, negative pictures, and no pictures). The last four conditions (further called 'deflation') were blocks with distentions (positive, neutral, negative pictures, and no pictures) in which the startle probes were delivered at times the balloon was in a deflated state. Next, the averaged raw startle magnitudes for each of these 11 conditions were standardized within participants (T-scores), so that they would be relative to each subjects' range of responding. Cells with averages that were based on less than 4 valid startle responses, were set as 'missing data'. This criterion was set to ensure reliable startle estimates for each of the eight inflation and deflation conditions, but resulted in $27.7 \%$ empty cells in our data matrix. Missing cells were not replaced with data from other cells.

Startle data were analyzed using a mixed regression model; this was done because such models provide a powerful and flexible approach to analyze repeated measures data with missing data cells (Blackwell, de Leon, \& Miller, 2006; Snijders \& Bosker, 2012; West, 2009). The model consisted of within-subject factors Picture (Neutral / Negative / Positive) and Context (Deflation / Inflation / Safe), and Gender (Male / Female) as between-subjects 
factor and their interactions. Although the experiment contained a seventh block with distentions but no pictures, we did not include that block in our startle analyses in order to allow for balanced post-hoc contrasts. We justify exclusion of this block, as prior analyses including this block, but requiring more complex models for analysis, result in the same main effects and interaction effects. (Inclusion of the no-picture block was originally intended to test for any differences between distentions accompanied by pictures, versus distentions without concurrent visual affective stimuli. No significant differences between picture and no picture distentions were found, so we did not include the picture free block in the final analysis of startle reported here.) The random part of the model consisted of a repeated measures effect with a first order autoregressive covariance structure with heterogeneous variances $\left(\mathrm{AR} 1_{(\text {Heterogeneous })}\right)\left(\mathrm{X}_{(7)}^{2}=31, p<.001\right)$ which had significantly better fit compared to its competitor (AR1). Significant interaction and main effects were explored through post hoc contrasts. Bonferroni corrections for multiple testing were applied. Data analysis was performed using SPSS 20.

3.3 Skin Conductance Levels. Mean absolute skin conductance levels (SCLs) were obtained per interval of 9 seconds. These SCLs were corrected for individual differences in response range by means of Rose's range correction (see Lykken and Venables, 1971). Next, a mean was calculated per block for each individual, and then a repeated measures ANOVA with gender as a between subject variable and Block (7 levels) as a within subject variable was performed on the obtained values.

3.4 Evaluative judgments. Self-reported fear, unpleasantness, and arousal scores of each individual for each block were also entered in a repeated measures ANOVA with gender as a between subject variable and Block (7 levels) as a within subject variable was performed. Tukey-Kramer post-hoc tests were used to follow-up main effects of Block. 
Greenhouse-Geisser corrections were applied where appropriate; we will report unadjusted degrees of freedom and adjusted $p$-values.

\section{Results}

An independent samples t-test indicated that thresholds of first pain did not differ significantly between male and female participants with mean distention volumes respectively $19 \mathrm{ml}(\mathrm{SD}=7 \mathrm{ml})$ and $17 \mathrm{ml}(\mathrm{SD}=7 \mathrm{ml})$.

4.1 Manipulation check. Following up the main effect of block, $F(6,132)=126.71$, $p<.001, \varepsilon=.64, \eta_{\mathrm{p}}{ }^{2}=.85$, confirmed that participants reported more intense pain for blocks with as compared to blocks without distentions, $F(6,132)=317.80, p<.001$. There were no significant main or interactions effects with gender.

The repeated measures ANOVA with position of the distention block as a within subject variable (1st, 3rd, 5th, or 7th) and gender as a between subject variable revealed no significant effects. However, the data showed a trend for women to report higher pain intensities for distention blocks than men (main effect of gender: $F(1,22)=4.13, p=.05$, $\left.\eta_{\mathrm{p}}{ }^{2}=.16\right)$. Also, the non-significant Gender $\mathrm{x}$ Block interaction suggested that men tended to habituate, whereas women tended to sensitize to visceral pain, $F(3,66)=2.12, p<.12, \varepsilon=$ $.85, \eta_{\mathrm{p}}^{2}=.09$

4.2 Eye blink startle. The mean startle magnitudes for each of the blocks (including the block without pictures) can be read from Table 2. There was a main effect of Context $F$ $(2,46.35)=5.26, p=.009$. Exploratory contrasts revealed higher startle amplitudes for deflation (when the balloon remained deflated in the distention blocks) relative to inflation $t$ $(55.8)=2.57, p=.039^{1}$ and the non-distention blocks $t(41.38)=3.24, p=.006^{1}$, whereas startle amplitudes did not differ during inflation compared to non-distension bocks $t(40.89)=$ 
$.36, p>.9^{1}$. In addition, this pattern depended on Gender indicated by the significant Context $\times$ Gender interaction $F(2,46.35)=4.77, p=.013$. Women, but not men showed startle potentiation during the distention blocks (inflation and deflation combined) relative to the non-distention blocks $\left(t(85.66)=4.22, p<.001^{2} ; t(78.01)=.05, p>.9^{2}\right)($ See Fig. 1). Women had startle potentiation during inflation $t(44.7)=2.65, p=.044^{2}$ ) and deflation $t$ $(43.93)=3.77, p=.002^{2}$ compared to the non-distention block, with no difference between deflation and inflation $t(56.68)=1.49, p=.56^{2}$. In contrast, for men no differences in startle were observed between inflation, deflation and non-distention blocks $\left(t(54.8)=2.1, p=.16^{2}\right.$; $\left.t(39.49)=1.19, p>.9^{2} ; t(38.55)=1.67, p=.41^{2}\right)$. Furthermore, there was no main effect of Picture $F(2,46.64)=.67, p=.51$, nor an interaction with Context $F(4,38.24)=.17, p=.95$, Gender $F(2,46.64)=.75, p=.48$ or between Context, Gender and Picture $F(4,38.25)=.61$, $p=.66$. Figure 1 displays the mean startle response for men and women during inflation, deflation and during non-distention, irrespective of picture viewing and picture content.

4.3 Skin Conductance Levels. Table 2 displays SCLs for each block. A repeated measures ANOVA indicated a main effect of block, $F(1,22)=3.07, p=.009, \varepsilon=.70, \eta_{\mathrm{p}}{ }^{2}=$ .12. Following-up the latter effect indicated that skin conductance was higher during blocks with, as compared to blocks without distentions, $F(1,22)=14.77, p<.001$. However, Tukey-Kramer post-hoc pairwise comparisons indicated that this was not true for each possible pair comparing a distention with a non-distention block (see Table 2). Furthermore, Tukey-Kramer tests yielded no evidence for picture content effects.

No other effects were observed for SCL.

4.4 Evaluative judgments. Self-reported fear revealed a main effect of gender with women reporting higher fear than men, $F(1,22)=8.57, p=.008, \varepsilon=.60, \eta_{\mathrm{p}}{ }^{2}=.28$, and a main effect of block, $F(6,132)=19.97, p<.001, \varepsilon=.60, \eta_{\mathrm{p}}{ }^{2}=.48$. Figure 2 displays the

\footnotetext{
${ }^{1}$ Bonferonni correction by factor 3

${ }^{2}$ Bonferonni correction by factor 4
} 
marginally significant Gender x Block interaction, $F(6,132)=2.44, p=.06, \varepsilon=.70, \eta_{\mathrm{p}}{ }^{2}=$ .09 , and suggests that with increasing levels of aversive stimulation (negative pictures and/or painful esophageal stimulation), women generally responded with a higher increase in fear compared to men.

For both pleasantness and arousal, only the main effects of block were significant, $F(6,132)=27.28, p<.001, \varepsilon=.57, \eta_{\mathrm{p}}{ }^{2}=.55$ and $F(6,132)=9.54, p<.001, \varepsilon=.57, \eta_{\mathrm{p}}{ }^{2}=$ .65 , respectively. Generally, Tukey-Kramer post-hoc comparisons (see Table 2) indicated that pleasantness was reduced for negative compared to positive and neutral picture viewing, and also reduced for blocks with compared to blocks without distentions. Furthermore, arousal ratings were influenced by picture content in the non-distention blocks (negative $>$ neutral, positive), but not in the distention blocks.

\section{Discussion}

The major aim of the current study was to investigate the modulation of eye blink startle elicited by aversive visceral stimulation. The limited number of studies using interoceptive stimuli to induce an unpleasant affective state found a potentiation of the startle blink only during anticipation of the aversive interoceptive stimulus (Hubbard et al., 2011; Lang et al., 2011; Melzig et al., 2008; Naliboff et al., 2009; Pappens et al., 2013; Twiss et al., 2009). However, any such potentiation of startle was absent when startles were elicited simultaneously with interoceptive stimulation (Ceunen, Vlaeyen, \& Van Diest, 2013; Pappens et al., 2010; 2012). Since most of these studies used respiratory or cold pain stimulation, it is uncertain whether these startle response findings apply to all interoceptive stimuli, or whether they are specific to these types of stimulation. To test the extent to which these findings can be generalized, we set up an experiment in which the pain stimulus was 
15 Startle responding in the context of visceral pain

purely visceral, without stimulation of overlying somatic tissues. To this end, we applied painful inflations of a small balloon in the distal esophagus.

The main finding of the present study is that women, but not men, showed a fearpotentiated startle during periods of time with intermittent painful distentions. Apart from a potentiated startle, also subjective fear and pain responses to the visceral pain stimulation were stronger in women compared to men, despite pain thresholds being individually determined prior to the protocol.

As distentions could not be presented for longer than 5 seconds due to the peristalsis of the esophagus pulling the inflated balloon, blocks labeled as 'distention blocks' contained a fair amount of time during which no distentions were actually present. This warranted further exploratory analyses wherein startle responses within these blocks were grouped into those occurring in the interval between two distentions (deflated), and those occurring simultaneous with distentions (inflated). It was found that in women, startles were potentiated during both deflated and inflated conditions relative to safe blocks, although this potentiation was strongest when balloons were deflated. Men showed no such startle potentiation.

These new findings add to the complex picture of startle responding during aversive interoceptive stimulation. Our findings in men are consistent with previous findings of our group on startle responding during respiratory stimuli and cold pain stimulation, during which no potentiation or even inhibition of startle was found (Ceunen et al., 2013; Pappens et al., 2010; 2012). However, the finding of an observed startle potentiation during inflation blocks in women, was unexpected. In previously published manuscripts, we have argued that either attentional mechanisms or threat imminence may play a role in startle response seen during interoceptive aversive stimulation. The threat imminence explanation holds that startle potentiation to aversive stimuli occurs only as long as no behavioral action is required - once a stimulus is so imminent that action is required or initiated, startle disappears or becomes 
inhibited (Lang et al., 1997; Low et al., 2008; Richter et al., 2012). However, our finding are not consistent with such explanation, because the lower fear reported by men than by women indicates that the absence of startle potentiation in men cannot be due to higher threat imminence in men.

The alternative explanation relates to attentional mechanisms, and holds that the presence of pain or dyspnea draws attention inwards and as a consequence reduces responsivity to extraneous stimuli such as the auditory startle probe (Alius et al., 2015; Ceunen et al., 2013; Deuter et al., 2012; Pappens et al., 2010). If we would apply this explanation to our findings, then this would imply that esophageal pain did not reduce attention to extraneous stimuli in the women in our study, whereas it did in the men. An argument in favor of women but not men being able to simultaneously process the startle probe and the visceral stimulus, is that women generally outperform men in multitasking (Jing et al., 2012; Mäntylä, 2013; Ren et al., 2009), although not always (Buser and Peter, 2012). Men on the other hand outperform women on monitoring accuracy (Mäntylä, 2013), and early research on visceral perception also suggests men are generally more accurate perceivers of interoceptive sensations (Pennebaker and Roberts, 1992). For our study, this then implies that perhaps men were solely focused on monitoring their internal state, and this prevented them from being responsive to the startle probe. This speculative mechanism could be further investigated, e.g., in experiments that manipulate the direction of attention (external/internal), or in experiments which measure event-related potentials to the startle probes and the balloon inflations.

A secondary aim of the present study was to investigate whether startle modulation by affect picture viewing would be different during times without pain stimulation compared to times with intermittent painful distentions. Despite effects of picture content on self-reported pleasantness, arousal and fear, the present study did not find modulation of the startle 
response by affective picture viewing. This may be due to the picture selection or the experimental context.

A limitation of the present study is that the current study used a rather complex design. As a general guideline for future studies on startle during interoceptive stimulation, it is recommendable to avoid designs that allow for higher order interactions that are difficult to interpret. A second limitation is that the current study was not specifically set up to study gender differences. Therefore, we need to reserve caution in interpreting the observed gender differences as being reflective of gender differences in visceral processing, until further studies replicate these gender effects. A third limitation is that we do not have information on esophageal compliance changes in response to the balloon distention, which means that we do not know whether there were individual differences in contraction force and degree of relaxation in response to stimulation. Any individual or gender related differences at this level could be also responsible for gender related differences in subjective experience and psychophysiological response measures.

In conclusion, the main finding of the present study is that in women, startle is potentiated during periods in which there is painful esophageal stimulation. We found that regardless of valence of the pictorial stimuli, participants reported higher fear, unpleasantness and arousal for the blocks with distentions, as compared to blocks without distentions. In women this was reflected in overall increased startle magnitude in the distention blocks, which is in accordance with the idea that startle is potentiated during arousing, negative affective states (Vrana et al., 1988). For both men and women, the distention blocks were more arousing than the ones without distention, which was evident from both increased electrodermal activity and increased self-reported arousal as compared to blocks without distention. 
As research on eyeblink startle in response to different types of interoceptive stimulation (and esophageal and other gastrointestinal sensations in particular) has just started recently, no conclusions can yet be drawn as to the underlying mechanism responsible for the difference between startle in response to esophageal stimulation as found in the current study, versus that to respiratory stimulation as found in previous studies (Ceunen et al., 2013; Pappens et al., 2010; 2013). Nevertheless, exploration, description, and replications of the typical startle response occurring during different types of interoceptive stimulation may help elucidate the differential responding upon interoceptive versus exteroceptive threat. The current results may contribute to a better understanding of (1) startle responding to different types of aversive interoceptive stimulation, and warrants further investigation of (2) possible gender differences in visceral pain processing. So far, both these directions of research have remained relatively unexplored, but we are confident they will receive more attention in future research.

\section{References}

Alius, M.G., Pané-Farré, C.A., Löw, A., Hamm, A.O., 2015. Modulation of the blink reflex and P3 component of the startle response during an interoceptive challenge. Psychophysiology 52, 140-148.

Aziz, Q., Thompson, D.G., Ng, V.W.K., Hamdy, S., Sarkar, S., Brammer, M.J., Bullmore, E.T., Hobson, A., Tracey, I., Gregory, L., Simmons, A., Williams, S.C.R., 2000. Cortical processing of human somatic and visceral sensation. The Journal of Neuroscience 20, 26572663.

Blumenthal, T.D., Cuthbert, B.N., Filion, D.L., Hackley, S., Lipp, O.V., Van Boxtel, A., 2005. Committee report: Guidelines for human startle eyeblink electromyographic studies. Psychophysiology 42, 1-15. 
Bradley, M.M., Codispoti, M., Cuthbert, B.N., Lang, P.J., 2001. Emotion and Motivation I: Defensive and Appetitive Reactions in Picture Processing. Emotion 1, 276-298.

Bradley, M.M., Lang, P.J., 1994. Measuring Emotion - the Self-Assessment Manikin and the Semantic Differential. Journal of Behavior Therapy and Experimental Psychiatry 25, 49-59. Bradley, M.M., Lang, P.J., 2000. Affective reactions to acoustic stimuli. Psychophysiology 37, 204-215.

Bradley, M.M., Lang, P.J., 2007. Emotion and Motivation, in: Cacioppo, J., Tassinary, L.G., Berntson, G.G. (Eds.), Handbook of Psychophysiology, 3 ed. Cambridge University Press, Cambridge, pp. 581-607.

Buser, T., Peter, N., 2012. Multitasking. Experimental Economics 15, 641-655.

Ceunen, E., Vlaeyen, J.W., Van Diest, I., 2013. Atypical modulation of startle in women in face of aversive bodily sensations. International Journal of Psychophysiology 88, 157-163. Coen, S.J., Yágüez, L., Aziz, Q., Mitterschiffthaler, M.T., Brammer, M., Williams, S.C.R., Gregory, L.J., 2009. Negative Mood Affects Brain Processing of Visceral Sensation. Gastroenterology 137, 253-261.

Craig, A.D.B., 2002. How do you feel? Interoception: the sense of the physiological condition of the body. Nature Reviews Neuroscience 3, 655-666.

Dawson, M.E., Schell, A.M., Filion, D.L., 2007. The Electrodermal System, in: Cacioppo, J.T., Tassinary, L.G., Berntson, G.G. (Eds.), Handbook of Psychophysiology, 3rd ed. Cambridge University Press, Cambridge, pp. 159-181.

De Clercq, A., Verschuere, B., De Vlieger, P., Crombez, G., 2006. Psychophysiological Analysis (PSPHA): A modular script-based program for analyzing psychophysiological data. Behavior Research Methods 38, 504-510.

Deuter, C.E., Kuehl, L.K., Blumenthal, T.D., Schulz, A., Oitzl, M.S., Schachinger, H., 2012. Effects of Cold Pressor Stress on the Human Startle Response. PLoS ONE 7, e49866. 
Dworkin, B.R., 2007. Interoception, in: Cacioppo, J.T., Tassinary, L.G., Berntson, G.G. (Eds.), Handbook of psychophysiology. Cambridge University Press, Cambridge.

Ehrlichman, H., Brown, S., Zhu, J., Warrenburg, S., 1995. Startle reflex modulation during exposure to pleasant and unpleasant odors. Psychophysiology 32, 150-154.

Horn, C., Schaller, J., Lautenbacher, S., 2012. Investigating the affective component of pain: No startle modulation by tonic heat pain in startle responsive individuals. International Journal of Psychophysiology 84, 254-259.

Hubbard, C.S., Ornitz, E., Gaspar, J.X., Smith, S., Amin, J., Labus, J.S., Kilpatrick, L.A., Rhudy, J.L., Mayer, E.A., Naliboff, B.D., 2011. Modulation of nociceptive and acoustic startle responses to an unpredictable threat in men and women. Pain 152, 1632-1640. Jansen, D.M., Frijda, N.H., 1994. Modulation of the acoustic startle response by film-induced fear and sexual arousal. Psychophysiology 31, 565-571.

Jing, Y., Jing, S., Huajian, C., Chuangang, S., Yan, L., 2012. The gender difference in distraction of background music and noise on the cognitive task performance, Natural Computation (ICNC), 2012 Eighth International Conference on. IEEE, pp. 584-587.

Kano, M., Farmer, A.D., Aziz, Q., Giampietro, V.P., Brammer, M.J., Williams, S.C., Fukudo, S., Coen, S.J., 2013. Sex differences in brain response to anticipated and experienced visceral pain in healthy subjects. American Journal of Physiology-Gastrointestinal and Liver Physiology 304, G687-G699.

Kilpatrick, L., Ornitz, E., Ibrahimovic, H., Treanor, M., Craske, M., Nazarian, M., Labus, J., Mayer, E., Naliboff, B., 2010. Sex-related differences in prepulse inhibition of startle in irritable bowel syndrome (IBS). Biological psychology 84, 272-278.

Labus, J.S., Gupta, A., Coveleskie, K., Tillisch, K., Kilpatrick, L., Jarcho, J., Feier, N., Bueller, J., Stains, J., Smith, S., Suyenobu, B., Naliboff, B., Mayer, E.A., 2013. Sex 
differences in emotion-related cognitive processes in irritable bowel syndrome and healthy control subjects. Pain 154, 2088-2099.

Lang, P.J., Bradley, M.M., Cuthbert, B.N., 1997. Motivated Attention: Affect, Activation, and Action, in: Lang, P.J., Simons, R.F., Balaban, M. (Eds.), Attention and Orienting: Sensory and Motivational Processes. Lawrence Erlbaum Associates, Inc., Mahway, New Jersey.

Lang, P.J., Bradley, M.M., Cuthbert, B.N., 2008. International affective picture system (IAPS): Affective ratings of pictures and instruction manual. Technical Report A-8. University of Florida, Gainesville, FL.

Lang, P.J., Wangelin, B.C., Bradley, M.M., Versace, F., Davenport, P.W., Costa, V.D., 2011. Threat of suffocation and defensive reflex activation. Psychophysiology 48, 393-396. Low, A., Lang, P.J., Smith, J.C., Bradley, M.M., 2008. Both Predator and Prey Emotional Arousal in Threat and Reward. Psychological Science 19, 865-873.

Lykken, D.T., Venables, P.H., 1971. Direct Measurement of Skin Conductance - Proposal for Standardization. Psychophysiology 8, 656-672.

Mäntylä, T., 2013. Gender differences in multitasking reflect spatial ability. Psychological science, 0956797612459660.

Melzig, C.A., Michalowski, J.M., Holtz, K., Hamm, A.O., 2008. Anticipation of interoceptive threat in highly anxiety sensitive persons. Behaviour Research and Therapy 46, $1126-1134$.

Merskey, H., Bogduk, N., 1994. Classification of Chronic Pain. IASP Press, Seattle. Mikels, J.A., Fredrickson, B.L., Larkin, G.R., Lindberg, C.M., Maglio, S.J., Reuter-Lorenz, P.A., 2005. Emotional category data on images from the international affective picture system. Behavior Research Methods 37, 626-630. 
Naliboff, B., Waters, A.M., Labus, J.S., Kilpatrick, L., Craske, M., Chang, L., Negoro, H., Ibrahimovic, H., Mayer, E.A., Ornitz, E., 2009. Increased Acoustic Startle Responses in IBS Patients During Abdominal and Non-Abdominal Threat. Journal of Urology 181, 2127-2133. Pappens, M., De Peuter, S., Vansteenwegen, D., Van den Bergh, O., Van Diest, I., 2012. Psychophysiological responses to CO2 inhalation. International Journal of Psychophysiology. Pappens, M., Van den Bergh, O., De Peuter, S., Bresseleers, J., Vansteenwegen, D., Van Diest, I., 2010. Defense reactions to interoceptive threats: A comparison between loaded breathing and aversive picture viewing. Biological Psychology 84, 98-103.

Pappens, M., Van den Bergh, O., Vansteenwegen, D., Ceunen, E., De Peuter, S., Van Diest, I., 2013. Learning to fear obstructed breathing: Comparing interoceptive and exteroceptive cues. Biological Psychology 92, 36-42.

Pennebaker, J.W., Roberts, T.-A., 1992. Toward a his and hers theory of emotion: Gender differences in visceral perception. Journal of Social and Clinical Psychology 11, 199-212. Ren, D., Zhou, H., Fu, X., 2009. A deeper look at gender difference in multitasking: Genderspecific mechanism of cognitive control, Natural Computation, 2009. ICNC'09. Fifth International Conference on. IEEE, pp. 13-17.

Rhudy, J.L., Meagher, M.W., 2001. The role of emotion in pain modulation. Current Opinion in Psychiatry 14, 241-245.

Richter, J., Hamm, A.O., Pané-Farré, C.A., Gerlach, A.L., Gloster, A.T., Wittchen, H.-U., Lang, T., Alpers, G.W., Helbig-Lang, S., Deckert, J., Fydrich, T., Fehm, L., Ströhle, A., Kircher, T., Arolt, V., 2012. Dynamics of Defensive Reactivity in Patients with Panic Disorder and Agoraphobia: Implications for the Etiology of Panic Disorder. Biological Psychiatry 72, 512-520.

Schupp, H.T., Cuthbert, B.N., Bradley, M.M., Birbaumer, N., Lang, P.J., 1997. Probe P3 and blinks: Two measures of affective startle modulation. Psychophysiology 34, 1-6. 
Smith, J.C., Bradley, M.M., Lang, P.J., 2005. State anxiety and affective physiology: effects of sustained exposure to affective pictures. Biological Psychology 69, 247-260.

Spruyt, A., Clarysse, J., Vansteenwegen, D., Baeyens, F., Hermans, D., 2010. Affect 4.0 A Free Software Package for Implementing Psychological and Psychophysiological Experiments. Experimental Psychology 57, 36-45.

Twiss, C., Kilpatrick, L., Craske, M., Buffington, C.A.T., Ornitz, E., Rodriguez, L.V., Mayer, E.A., Naliboff, B.D., 2009. Increased Startle Responses in Interstitial Cystitis: Evidence for Central Hyperresponsiveness to Visceral Related Threat. Journal of Urology 181, 2127-2133. Vrana, S.R., Spence, E.L., Lang, P.J., 1988. The startle probe response: a new measure of emotion? Journal of Abnormal Psychology 97, 487-491.

Wiech, K., Tracey, I., 2009. The influence of negative emotions on pain: Behavioral effects and neural mechanisms. Neuroimage 47, 987-994.

World Medical Association, 1997. World Medical Association Declaration of Helsinki Recommendations guiding physicians in biomedical research involving human subjects Adopted by the 18th World Medical Assembly Helsinki, Finland, June, 1964. Cardiovascular Research 35, 2-3. 


\section{Author Notes}

Erik Ceunen and Jonas Zaman were supported by a grant from the Fund for Scientific Research, Flanders (FWO, Vlaanderen). Lukas Van Oudenhove is a research professor of the KU Leuven Special Research Fund and a postdoctoral research fellow of the Fund for Scientific Research - Flanders (FWO - Vlaanderen). 


\section{Appendix}

The following pictures were selected from the International Affective Picture System (IAPS): Positive pictures, block A: 1463, 1603, 1620, 1731, 2058, 2209, 2216, 2299, 2311, 2340, $2341,2345,2388,2395,2398,2501,2550,4532,4610,4614,5001,5201,5260,5480,5551$, $5621,5623,5760,5994,7230,7282,7325,8370,8461,8470,8499$

Positive pictures, block B: 1340, 1710, 1750, 1920, 2165, 2304, 2360, 2387, 2530, 2598, $2660,4574,4622,4626,4640,5010,5700,5811,5831,5833,5836,5849,7280,7340,7502$, $7580,8162,8170,8185,8210,8380,8420,8496,8497,8502,8540$

Neutral pictures, block A: 1121, 1560, 1670, 1850, 2025, 2038, 2104, 2190, 2191, 2206, $2210,2214,2235,2272,2305,2381,2393,2396,2397,2435,2480,2485,2514,2579,2580$, $2597,5395,5455,5520,7002,7004,7036,7140,7205,7495,7640$

Neutral pictures, block B: 1675, 1942, 1947, 2102, 2200, 2357, 2372, 2383, 2385, 2445, $2487,2495,2499,2518,2575,2593,2594,2850,2870,2880,2980,5471,5740,7037,7041$, 7130, 7217, 7491, 7493, 7496, 7504, 7506, 7546, 7550, 8211, 8311

Negative pictures, block A: 1114, 1302, 2095, 2120, 2683, 2691, 2692, 2694, 2703, 2751, $2800,2811,3500,3530,4621,5971,6020,6190,6212,6242,6312,6313,6560,6838,6940$, $8485,9001,9050,9140,9270,9340,9342,9409,9423,9600,9900$

Negative pictures, block B: 1200, 1932, 2799, 2900, 4635, 5973, 6241, 6250, 6315, 6370, 6550, 6571, 6800, 6821, 6840, 9006, 9041, 9181, 9220, 9230, 9404, 9410, 9417, 9419, 9421, 9424, 9425, 9426, 9429, 9440, 9470, 9520, 9561, 9622, 9800, 9911. 
26 Startle responding in the context of visceral pain

Table 1. Overview of the 11 within subject conditions.

\begin{tabular}{ccccc}
\hline Context & Pictures & Block & Condition \\
\hline $\begin{array}{c}\text { 'Safe' } \\
\text { (distention-free } \\
\text { blocks) }\end{array}$ & Positive & 1 & 1 \\
\hline & Neutral & 2 & & 2 \\
& Positive & 4 & Deflation & 4 \\
& & 3 & Inflation & 5 \\
& Neutral & 5 & Deflation & 6 \\
Blocks with & & & Inflation & 7 \\
distentions & Negative & 6 & Deflation & 8 \\
& & & Inflation & 9 \\
& & & Deflation & 10 \\
& No pictures & 7 & Inflation & 11 \\
\hline
\end{tabular}


Table 2. Means (standard deviation) of skin conductance levels, startle magnitudes, and selfreported fear, pain intensity, pleasantness and arousal for each of the seven blocks.

\begin{tabular}{|c|c|c|c|c|c|c|c|}
\hline & \multicolumn{3}{|c|}{ Non-distention Blocks } & \multicolumn{4}{|c|}{ Distention Blocks } \\
\hline & Pos pic & Neu pic & Neg pic & Pos pic & Neu pic & Neg pic & No pic \\
\hline \multirow[t]{2}{*}{ SCL } & $0.42_{\mathrm{ab}}$ & $0.46_{\mathrm{ab}}$ & $0.39 \mathrm{a}$ & $0.51_{\mathrm{b}}$ & $0.48_{\mathrm{ab}}$ & $0.53_{b}$ & $0.52_{b}$ \\
\hline & (0.19) & $(0.17)$ & $(0.18)$ & $(0.18)$ & (0.19) & $(0.18)$ & $(0.15)$ \\
\hline \multirow[t]{2}{*}{ Startle } & 46.80 & 48.24 & 50.01 & 51.41 & 51.64 & 52.86 & 52.75 \\
\hline & $(5.31)$ & $(6.66)$ & $(6.31)$ & $(8.88)$ & $(10.81)$ & $(9.06)$ & $(13.65)$ \\
\hline Pain & $0.75_{\mathrm{a}}$ & $0.71_{\mathrm{a}}$ & $0.50_{\mathrm{a}}$ & $5.96_{b}$ & $6.25_{b}$ & $6.33_{\mathrm{b}}$ & $6.63_{b}$ \\
\hline intensity & $(1.33)$ & $(1.57)$ & $(1.10)$ & $(1.57)$ & $(1.80)$ & $(1.63)$ & (1.38) \\
\hline \multirow[t]{2}{*}{ Fear } & $0.71_{\mathrm{a}}$ & $0.96_{\mathrm{a}}$ & $2.58_{b}$ & $2.86_{b c}$ & $3.58 \mathrm{bc}$ & $3.96_{c}$ & $3.75_{\mathrm{c}}$ \\
\hline & $(0.91)$ & $(1.60)$ & $(2.32)$ & $(2.15)$ & $(2.50)$ & $(2.54)$ & $(2.56)$ \\
\hline \multirow[t]{2}{*}{ Pleasantness } & $6.92_{\mathrm{a}}$ & $5.96_{a}$ & $4.25_{\mathrm{b}}$ & $4.09_{b}$ & $3.83_{\mathrm{bc}}$ & $2.75_{\mathrm{c}}$ & $2.88_{c}$ \\
\hline & (1.61) & $(1.49)$ & $(1.57)$ & $(1.77)$ & $(1.43)$ & (1.07) & $(0.99)$ \\
\hline \multirow[t]{2}{*}{ Arousal } & $3.33_{\mathrm{a}}$ & $3.17 \mathrm{a}$ & $4.33_{b}$ & $4.82_{b c}$ & $5.33 \mathrm{bc}$ & $5.58_{c}$ & $5.25_{\mathrm{bc}}$ \\
\hline & $(1.83)$ & $(1.37)$ & (1.83) & $(1.46)$ & (1.83) & (1.53) & (1.59) \\
\hline
\end{tabular}

Note. Pos pic=positive pictures; Neg pic=negative pictures; Neu pic=neutral pictures; No pic=without pictures; SCL= Range-corrected Skin Conductance Level; Startle $=$ T-score of the Startle Blink Magnitude; Pain intensity (0-10 scale); Fear (0-10 scale); Pleasantness (1=unpleasant; 9=pleasant); Arousal (1=calm; 9=aroused). Means in the same row which share a subscript are not significantly different from one another according to Tukey-Kramer post-hoc tests $(p<.05)$. 


\section{Figure Captions}

Fig. 1. Startle amplitudes (mean T-scores + SE) during deflation and inflation (distention blocks) and during safe (non-distention) blocks for women and men. ${ }^{*} \mathrm{p}=.002,{ }^{*} \mathrm{p}<.05$. Fig. 2. Self-reported fear (mean $\pm \mathrm{SE}$, on a 0-10 scale) of women and men for the seven blocks in the experiment. 
Table 1. Overview of the 11 within subject conditions.

\begin{tabular}{ccccc}
\hline Context & Pictures & Block & Condition \\
\hline $\begin{array}{c}\text { 'Safe' } \\
\text { (distention-free } \\
\text { blocks) }\end{array}$ & Positive & 1 & 1 \\
& Neutral & 2 & & 2 \\
& Positive & 4 & Deflation & 4 \\
& & 3 & Inflation & 5 \\
& Neutral & 5 & Deflation & 6 \\
Blocks with & & & Inflation & 7 \\
distentions & Negative & 6 & Deflation & 8 \\
& & & Inflation & 9 \\
& & 7 & Deflation & 10 \\
& No pictures & & Inflation & 11 \\
\hline
\end{tabular}


Table 2. Means (standard deviation) of skin conductance levels, startle magnitudes, and selfreported fear, pain intensity, pleasantness and arousal for each of the seven blocks.

\begin{tabular}{|c|c|c|c|c|c|c|c|}
\hline & \multicolumn{3}{|c|}{ Non-distention Blocks } & \multicolumn{4}{|c|}{ Distention Blocks } \\
\hline & Pos pic & Neu pic & Neg pic & Pos pic & Neu pic & Neg pic & No pic \\
\hline \multirow[t]{2}{*}{ SCL } & $0.42_{\mathrm{ab}}$ & $0.46_{\mathrm{ab}}$ & $0.39 \mathrm{a}$ & $0.51_{b}$ & $0.48_{\mathrm{ab}}$ & $0.53 \mathrm{~b}$ & $0.52_{\mathrm{b}}$ \\
\hline & (0.19) & $(0.17)$ & $(0.18)$ & $(0.18)$ & (0.19) & $(0.18)$ & $(0.15)$ \\
\hline \multirow[t]{2}{*}{ Startle } & 46.80 & 48.24 & 50.01 & 51.41 & 51.64 & 52.86 & 52.75 \\
\hline & $(5.31)$ & $(6.66)$ & $(6.31)$ & $(8.88)$ & $(10.81)$ & $(9.06)$ & $(13.65)$ \\
\hline Pain & $0.75_{\mathrm{a}}$ & $0.71_{\mathrm{a}}$ & $0.50_{\mathrm{a}}$ & $5.96 \mathrm{~b}$ & $6.25_{b}$ & $6.33_{b}$ & $6.63 \mathrm{~b}$ \\
\hline intensity & $(1.33)$ & $(1.57)$ & $(1.10)$ & $(1.57)$ & $(1.80)$ & (1.63) & $(1.38)$ \\
\hline \multirow[t]{2}{*}{ Fear } & $0.71_{\mathrm{a}}$ & $0.96_{\mathrm{a}}$ & $2.58_{b}$ & $2.86_{b c}$ & $3.58_{\mathrm{bc}}$ & $3.96_{\mathrm{c}}$ & $3.75_{\mathrm{c}}$ \\
\hline & $(0.91)$ & $(1.60)$ & $(2.32)$ & $(2.15)$ & $(2.50)$ & $(2.54)$ & $(2.56)$ \\
\hline \multirow[t]{2}{*}{ Pleasantness } & $6.92_{\mathrm{a}}$ & $5.96_{a}$ & $4.25_{\mathrm{b}}$ & $4.09_{b}$ & $3.83_{\mathrm{bc}}$ & $2.75_{\mathrm{c}}$ & $2.88_{\mathrm{c}}$ \\
\hline & (1.61) & (1.49) & $(1.57)$ & (1.77) & $(1.43)$ & $(1.07)$ & $(0.99)$ \\
\hline \multirow[t]{2}{*}{ Arousal } & $3.33_{\mathrm{a}}$ & $3.17_{\mathrm{a}}$ & $4.33_{b}$ & $4.82_{\mathrm{bc}}$ & $5.33_{\mathrm{bc}}$ & $5.58_{c}$ & $5.25_{\mathrm{bc}}$ \\
\hline & $(1.83)$ & $(1.37)$ & $(1.83)$ & $(1.46)$ & (1.83) & (1.53) & (1.59) \\
\hline
\end{tabular}

Note. Pos pic=positive pictures; Neg pic=negative pictures; Neu pic=neutral pictures; No pic=without pictures; SCL= Range-corrected Skin Conductance Level; Startle $=\mathrm{T}$-score of the Startle Blink Magnitude; Pain intensity (0-10 scale); Fear (0-10 scale); Pleasantness (1=unpleasant; $9=$ =pleasant); Arousal (1=calm; $9=$ =aroused $).$ Means in the same row which share a subscript are not significantly different from one another according to Tukey-Kramer post-hoc tests $(p<.05)$. 


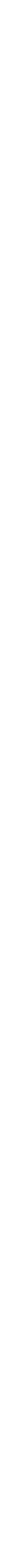




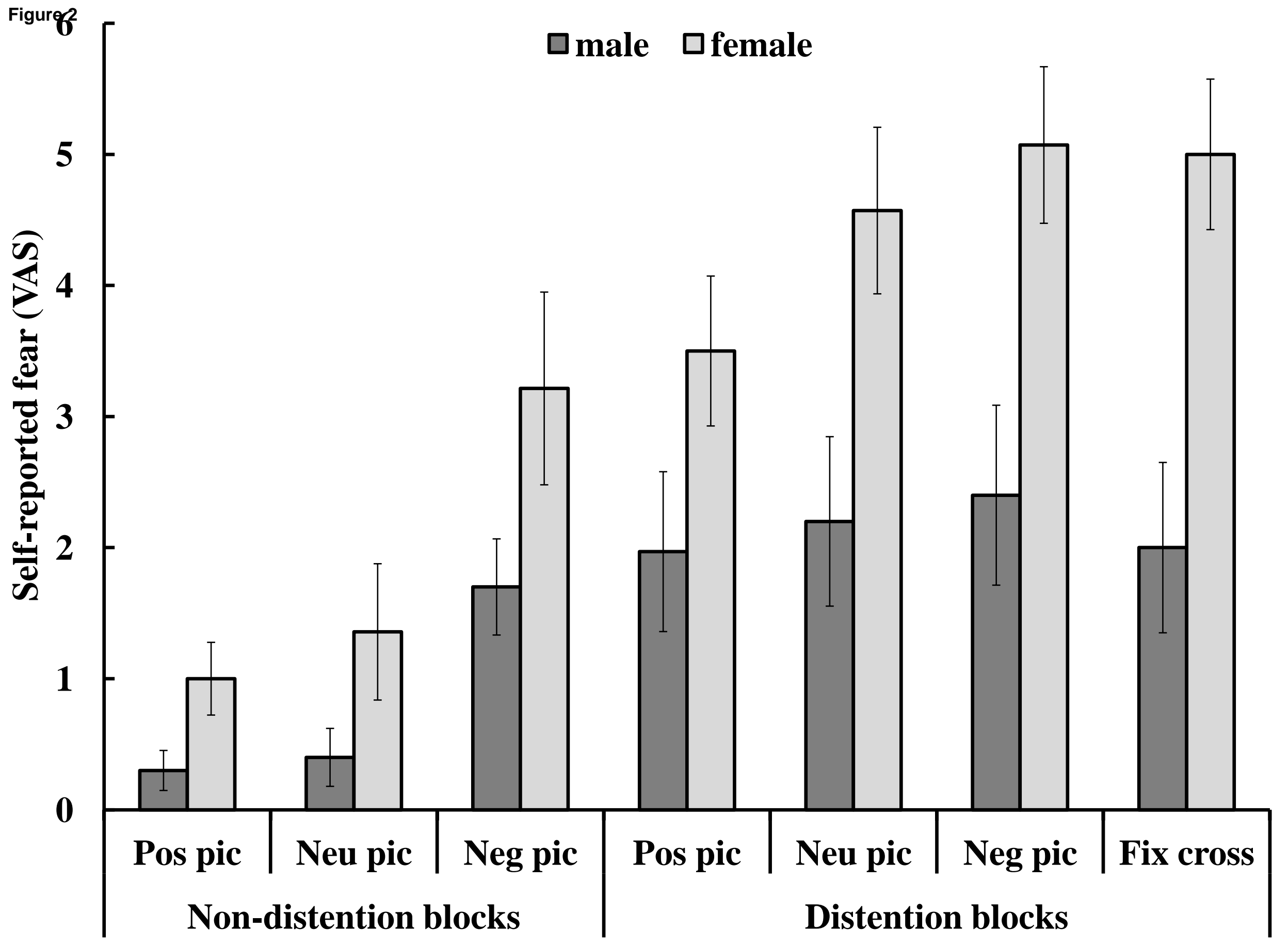

